\title{
ANTIDEPRESSIVOS TRICÍCLICOS: REVISÃO LITERÁRIA SOBRE SUAS INDICAÇÕES, CONTRAINDICAÇÕES E POSSÍVEIS EFEITOS ADVERSOS
}

\author{
Bárbara de Freitas Lopes*1, Luana Kárem Ferreira de Souza Ribeiro², Andressa Dias de \\ Andrade Fontes Amorim ${ }^{3}$, Ivan Gilson Silva Moura ${ }^{4}$, Mariane Costa Santos de Tavares ${ }^{5}$, Bruna \\ Batistone Bertachi6, Vítor de Freitas Lopes7, Caroline Braga Palacio8, Thaiara Lana Chagas \\ Cardoso $^{9}$, Pedro Marcos Alves Medeiros ${ }^{9}$, Rosy Aline Lopes de Oliveira ${ }^{9}$ \\ and Maria Clara Pena Ferraz ${ }^{10}$
}

\begin{abstract}
${ }^{1}$ Discente do Curso de Graduação em Medicina. Faculdades Santo Agostinho, Vitória da Conquista - BA; ${ }^{2}$ Médica. Docente do Curso de Graduação em Medicina. Faculdades Santo Agostinho, Vitória da Conquista - BA; ${ }^{3}$ Médica. Docente do Curso de Graduação em Medicina. Faculdades Santo Agostinho, Vitória da Conquista - BA; ${ }^{4}$ Médico Psiquiatra. Docente do Curso de Graduação em Medicina. Faculdades Santo Agostinho, Vitória da Conquista - BA; ${ }^{5}$ Fisioterapeuta. Especialista em Terapia Intensiva e Deficiências múltiplas e sensoriais. Discente do Curso de Graduação em Medicina. Faculdades Santo Agostinho, Vitória da Conquista - BA; ${ }^{6}$ Médica. Especialista em Atenção Básica pela Universidade Federal do Paraná - UFPR; ${ }^{7}$ Médico. Hospital São Vicente de Paulo, Vitória da Conquista - BA; ${ }^{8}$ Médica. DSEI Alto Rio Solimões, Pólo Base Belém do Solimões; ${ }^{9}$ Discente do Curso de Graduação em Medicina. Faculdades Santo Agostinho, Vitória da Conquista - BA; ${ }^{10}$ Discente do Curso de Graduação em Medicina. Faculdades Santo Agostinho, Itabuna - BA
\end{abstract}

\section{ARTICLE INFO}

\section{Article History:}

Received $11^{\text {th }}$ January, 2021

Received in revised form

$16^{\text {th }}$ February, 2021

Accepted $03^{\text {rd }}$ March, 2021

Published online $22^{\text {th }}$ April, 2021

\section{Key Words:}

Antidepressivos. Antidepressivos Tricíclicos. Depressão. Dependência medicamentosa.

Psicofarmacologia.

*Corresponding author:

Bárbara de Freitas Lopes

\begin{abstract}
Objetivo: Verificar o impacto do tratamento com os antidepressivos tricíclicos (ADTs) nos usuários, abordando o mecanismo de ação, indicações e contraindicações, bem como possíveis interações medicamentosas e efeitos colaterais desses fármacos. Métodos: Realiza-se, então, revisão integrativa de literatura nas bases de dados da US National Library of Medicina (Pubmed), Scientific Eletronic Library Online (SciELO) e da Literatura Latino-Americana e do Caribe em Ciências da Saúde (Lilacs). Considerando artigos científicos na língua portuguesa e inglesa publicados entre 1999 a 2020. Resultados: Vinte e dois estudos foram selecionados para análise e discussão dos dados, de acordo com os critérios de busca e seleção identificados. Consideraçõesfinais: Os antidepressivos tricíclicos são medicamentos amplamente utilizados na clínica médica devido ao custo benefício que apresentam. Entretanto, a presente revisão também sugere que os ADTs possuem um alto risco toxicológico e diversos efeitos colaterais, presentes, especialmente, quando utilizados em idosos ou em pacientes com comorbidades, em doses altas ou de forma concomitante com outros medicamentos, por possuírem uma significativa interação medicamentosa com diversas classes de remédios. Logo, vale ressaltar que essas reações adversas podem ser minimizadas ou até mesmo evitadas, desde que os medicamentos sejam utilizados de maneira cautelosa, com monitoramento constante dos pacientes e nas doses mais baixas possíveis para se obter o efeito desejado com o mínimo de efeitos colaterais.
\end{abstract}

Copyright (C) 2021, Bárbara de Freitas Lopes et al, This is an open access article distributed under the Creative Commons Attribution License, which permits unrestricted use, distribution, and reproduction in any medium, provided the original work is properly cited.

Citation: Bárbara de Freitas Lopes, Luana Kárem Ferreira de Souza Ribeiro, Andressa Dias de Andrade Fontes Amorim, Ivan Gilson Silva Moura, Mariane Costa Santos de Tavares, Bruna Batistone Bertachi6, Vitor de Freitas Lopes, Caroline Braga Palacio, Thaiara Lana Chagas Cardoso, Pedro Marcos Alves Medeiros, Rosy Aline Lopes de Oliveira and Maria Clara Pena Ferraz, 2021. "Atendimento aos dependentes quimicos no brasil: histórico e reflexão", International Journal of Development Research, 11, (04), 46244-46247.

\section{INTRODUÇÃO}

Nos últimos anos percebe-se uma crescente exigência para a obtenção de sucesso nos âmbitos da vida profissional e financeira e como consequência, houve um impacto negativo na atenção dada à saúde mental dos indivíduos que passaram a ter menos tempo para se dedicar à manutenção da saúde psíquica e à relações interpessoais de qualidade afetiva. Logo, na realidade contemporânea houve um aumento no número de pessoas financeiramente independentes e bem-sucedidas no ambiente de profissional, mas isoladas e emocionalmente instáveis, o que acabou por desencadear uma elevação significativa no número de transtornos depressivos (LOPES; FAERSTEIN; CHOR, 2003). Vale destacar que entre esses transtornos está a depressão, a qual é conhecida pela presença de 
alguns sintomas, tais como apatia, irritabilidade, perda de interesse, tristeza, atraso motor ou agitação, ideias agressivas, desolação e múltiplas queixas somáticas como insônia, fadiga e anorexia (ESTEVES; GALVAN, 2006). Logo, o consumo de antidepressivos tem aumentado significativamente na última década, pois tem ocorrido um maior diagnóstico das doenças depressivas e surgiram novas medicações, além do fato de que esses remédios têm tido suas indicações terapêuticas ampliadas (RODRIGUES; FACCHINI; LIMA, 2006). Quanto à ampla ocorrência de distúrbios psiquiátricos, os fatores ambientais estão direta e indiretamente relacionados ao aparecimento e ao agravamento distúrbios psiquiátricos, como por exemplo a elevada ocorrência de eventos estressantes durante a vida, a crescente urbanização e industrialização, desigualdade social e desigualdade de acesso aos serviços de saúde, desemprego, violência seja física seja emocional e má condições de moradia (LOPES; FAERSTEIN; CHOR, 2003). Dessa forma, diariamente, surgem novos tratamentos, alternativos ou tradicionais, que buscam tratar transtornos afetivos e emocionais como os quadros depressivos. Vale dizer, que como consequência o consumo de antidepressivos tem aumentado significativamente na última década, pois, como já foi dito, tem ocorrido um maior diagnóstico das doenças depressivas e surgiram novas medicações, além do fato de que esses remédios têm tido suas indicações terapêuticas ampliadas (RODRIGUES; FACCHINI; LIMA, 2006). É relevante salientar que os antidepressivos são utilizados predominantemente por mulheres, sendo que estatisticamente a diferença no número de pacientes do sexo feminino e masculino que utilizam antidepressivos é significativa (ROCHA; WERLANG, 2013).

A descoberta dos medicamentos antidepressivos se deu no fim da década de 50, trazendo um grande avanço para o tratamento dos transtornos depressivos já que eles se tornaram passiveis de tratamento assim como as doenças crônicas. Apenas duas classes medicamentosas de antidepressivos existiam até a década de 80 , os tricíclicos (ADTs) e os inibidores da monoaminooxidase (IMAOs). Posteriormente descobriu-se os inibidores da receptação da serotonina (ISRSs), os quais são mais seletivos e portanto, possuem menos efeitos adversos (MORENO; MORENO; SOARES, 1999). Os antidepressivos são medicamentos utilizados para tratar especialmente as doenças psiquiátricas e são classificados de acordo com sua estrutura química e suas propriedades farmacológicas. Os antidepressivos heterocíclicos (tricíclicos e tetracíclicos) possuem estrutura cíclica (anéis benzênicos), e os tricíclicos são divididos em aminas terciárias (imipramina, amitriptilina, trimipramina e doxepina) e as aminas secundárias (desmetilimipramina, nortriptilina e protriptilina). É importante relatar que a maprotilina e a amoxapina são antidepressivos tetracíclicos, pois possuem características farmacológicas semelhantes (MORENO; MORENO; SOARES, 1999). Acredita-se que a eficácia dos antidepressivos tricíclicos se deva ao aumento de neurotransmissores disponíveis no sistema nervoso central (SNC) associado à redução do número de neuroreceptores, com consequente aumento de sua sensibilidade. Os ADTs levam de maneira aguda ao aumento na eficiência da transmissão monoaminérgica (e possivelmente GABAérgica), além de envolverem os sistemas serotoninérgico e noradrenérgico. Por meio deste mecanismo, elevam a concentração sináptica tanto de norepinefrina quanto de serotonina por meio da inibição do metabolismo, ao bloquear a captura neuronal ou atuando em auto receptores pré-sinápticos (MORENO; MORENO; SOARES, 1999). Também atuam bloqueando os receptores histaminérgicos, alfa adrenérgicos e muscarínicos, inibindo a ATPase de sódio (NA+), e potássio $(\mathrm{K} .+)$. O bloqueio desses canais resulta na estabilização das membranas, bloqueando os canais de cálcio dependentes da voltagem, antagonizando o receptor de N-metil-D-aspartato (NMDA) e se ligando a receptores opióides endógenos (SINDRUP et al., 2005).

Ademais, o consumo de antidepressivos cresceu em todo o mundo nas últimas três décadas' sendo que em alguns países, os antidepressivos se tornaram a droga psicotrópica mais prescrita(VICENTE et al., 2015). Assim, este trabalho objetiva analisar as informações obtidas acerca dos antidepressivos tricíclicos e assim, consequentemente, trazer informações sobre os impactos adversos ao tratamento com os antidepressivos tricíclicos levando em consideração seu mecanismo de ação, suas indicações e interações medicamentosas e por fim, seus efeitos colaterais.

\section{METÓDOS}

O estudo caracteriza-se como uma revisão Integrativa da Literatura Para o levantamento dos artigos na literatura, realizou-se uma busca nas bases de dados da US National Library of Medicina (Pubmed), Scientific Eletronic Library Online (SciELO) e da Literatura LatinoAmericana e do Caribe em Ciências da Saúde (Lilacs). Foram utilizados, para busca dos artigos, os seguintes descritores em saúde e suas combinações em língua portuguesa e inglesa: Antidepressivos; Antidepressivos Tricíclicos; Depressão; Dependência medicamentosa; Psicofarmacologia. Foram considerados elegíveis para inclusão na revisão estudos publicados em inglês e português entre os anos de 1999 e 2020, que relacionassem o impacto dos efeitos adversos ao tratamento com antidepressivos tricíclicos à sua ampla utilização no âmbito da saúde. Foram excluídos, na análise integrativa dos artigos, estudos referentes a artigos não-originais (editoriais, comentários, revisões, capítulos de livros e cartas) e artigos que apresentavam duplicidade em diferentes bases de dados.

A primeira fase da seleção foi caracterizada pela análise dos títulos, posteriormente, foi realizada a análise dos resumos. Por último, foi realizada a leitura integral dos estudos para verificar os critérios de elegibilidade. Haja vista que esse trabalho se trata de uma pesquisa observacional, do tipo revisão integrativa de estudos já publicados na literatura, dispensou-se a submissão ao comitê de ética em pesquisa em seres humanos, dado que as informações utilizadas são secundárias e já foram divulgados. A pesquisa foi conduzida observando a Resolução 466/12 CONEP-CNS/MS. Os resultados da pesquisa serão divulgados no Internacional Journal of Development Research. A pesquisa possibilitará a ampliação do conhecimento do tema estudado, podendo impactar na saúde dos pacientes que fazem uso dos antidepressivos tricíclicos e na prescrição destes medicamentos, uma vez que os profissionais terão acesso a um conhecimento que objetiva que a utilização desses remédios ocorra de maneira mais cuidadosa e criteriosa, tendo em vista que eles terão informações acerca dos efeitos colaterais que mais comumente ocorrem, além da correlação desses efeitos adversos com o mecanismo de ação e quais são os grupos de pessoas que estão mais suscetíveis ao surgimento dessas reações adversas.

\section{RESULTADOS E DISCUSSÃO}

Os ADTs, são exemplos de drogas atualmente prescritas de maneira indiscriminada, pois possuem um baixo custo e larga distribuição pelo sistema único de saúde (SUS), além de serem fármacos de fácil acesso, embora eles constituam uma classe de drogas que possui alto risco toxicológico, visto que seu uso abusivo desencadeia diversos efeitos colaterais podendo até mesmo representar risco de morte. Essa enorme utilização pelo SUS se dá devido ao fato de que o Brasil não é um país com muitos recursos, o que faz com que os medicamentos fornecidos gratuitamente sejam prescritos pela disponibilidade e não por serem os medicamentos mais adequados (SEBASTIÃO; PELÁ, 2004). Os antidepressivos tricíclicos (ADT) são largamente utilizados não apenas no tratamento de quadros depressivos, mas possuem aplicação também em quadros de dores crônicas, como lombalgias e no tratamento de dores neuropáticas, o que é explicitado pelas diretrizes para o tratamento da dor neuropática que consideram como primeira linha: os anticonvulsivantes (gabapentina e pregabalina), os antidepressivos tricíclicos (amitriptilina, imipramina, clomipramina e nortriptilina), supracitados, e os inibidores seletivos da receptação de serotonina e de noradrenalina, tais como a duloxetina e a venlafaxina (STUMP; KOBAYASHI; CAMPOS, 2016). Segundo Cunha, Barrucand e Verçosa (2009) as medicações do tipo ADT vem sendo cada vez mais prescritas para pacientes com dores neuropáticas, 
fibromialgia, cólon irritável, com quadros de cefaleias tipo migrânea e cervicogênica e quando apresentam dores miofasciais crônicas". Vale destacar que a amitriptilina e a ciclobenzaprina são recomendadas no tratamento da FM nas diretrizes de 2016 da Liga Europeia Contra o Reumatismo (European League Against Rheumatism ou EULAR) e da Sociedade Canadense de Dor (Canadian Pain Society ou CPS), de 2013 (OLIVEIRA JÚNIOR; ALMEIDA, 2018). Oliveira et al. (2016) traz que a nortriptilina também pode ser utilizada de maneira apropriada para tratar a doença de Parkinson quando associada à depressão em idosos jovens. E, por fim, a amitriptilina é um antidepressivo tricíclico efetivamente utilizado na profilaxia da enxaqueca, independentemente de sua atividade antidepressiva e mostrou-se superior ao Sistema de Supressão de Tensão de Inibição do Trigêmeo Nociceptivo (NTI-tss) e à placa não oclusal (BRUNO; KRYMCHANTOWSKI, 2018).

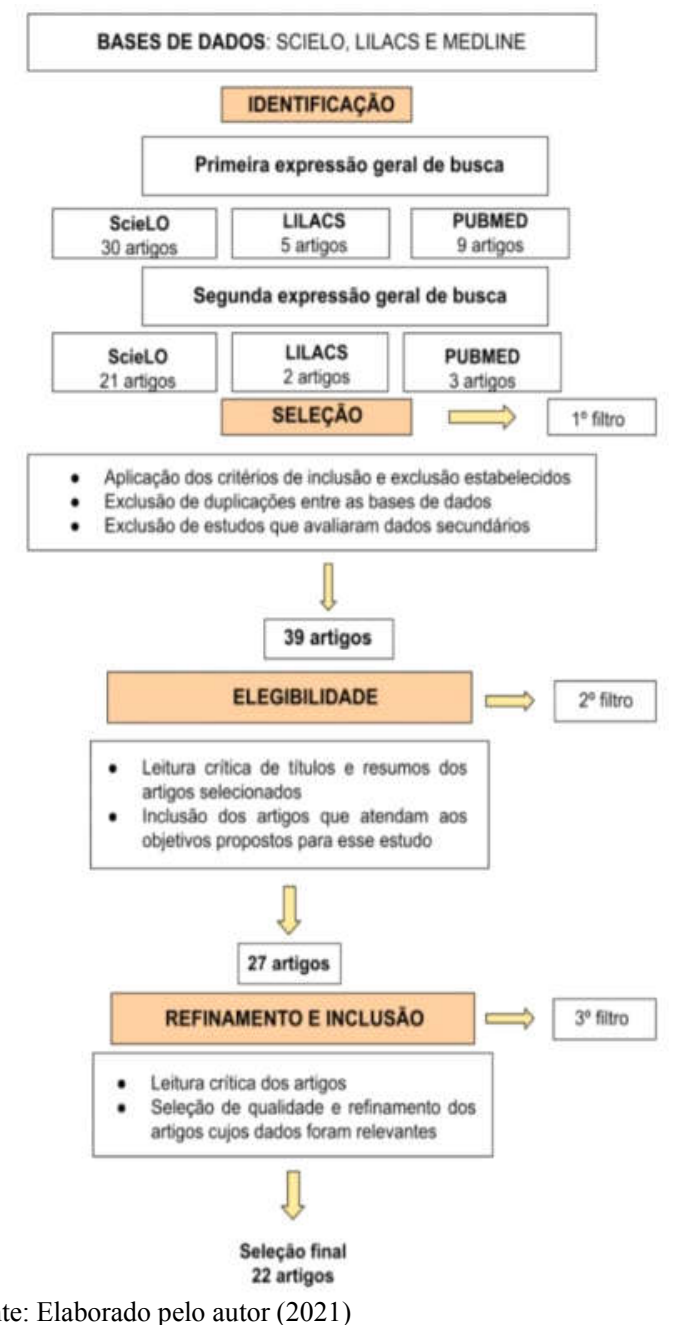

Fonte: Elaborado pelo autor (2021)

\section{Figura 1. Fluxograma de seleção e inclusão dos artigos}

Logo, como já foi dito o uso dos antidepressivos tricíclicos desencadeiam um bloqueio nos receptores histamínicos, colinérgicos e alfa-adrenérgicos, acarretando efeitos colaterais moderados à graves e alto risco de toxicidade, devendo por este motivo ser muito bem avaliados, sendo que os efeitos colaterais mais comuns são no trato gastrointestinal, pois as células gastrointestinais usam mais serotonina do que os demais órgãos do corpo humano. No mais, os efeitos colaterais, principalmente os relacionados à ação anticolinérgica, são notados já nas primeiras horas de uso da medicação, tais efeitos são comumente os responsáveis pelo grande número de abandono do tratamento nas primeiras semanas de utilização da medicação (MORENO; MORENO; SOARES, 1999). Outros efeitos colaterais são relatados, entre os quais estão: cefaléia, incoordenação, transtorno do sono, disfunção sexual e tremor, sendo que alguns desses efeitos são transitórios e acabam desaparecendo após um tempo de uso (LOPES, 2015). Segundo Scalco (2002), os ADTs ainda podem causar hipotensão ortostática, mioclonias, convulsão, visão turva, boca seca,taquicardia e prolongamento dos intervalos PR e QRS no ECG, constipação crônica, delirium, diminuição da contratilidade cardíaca, além de efeitos cognitivos, em doentes idosos e com déficits cognitivos preexistentes. Santana e Góis (2013) afirmam que os medicamentos antidepressivos tricíclicos possuem uma série de efeitos deletérios que muitas vezes são ignorados, que estão relacionados à diversos casos de tentativas de suicídio, pois tais medicamentos em doses elevadas podem desencadear reações tóxicas como a rabdomiólise e são medicamentos de fácil acesso. A amitriptilina, por exemplo, já foi relacionada como potencial causadora de alterações eletrocardiográficas em pacientes com depressão, outros achados indicam que sua utilização associada à anticonvulsivantes favorece o aparecimento de convulsões (CAMPIGOTTO, 2008; CUNHA; BARRUCAND; VERÇOSA, 2009). Este maior controle é necessário visto que o ato de se automedicar é uma prática muito comum no ambiente social, não apenas no Brasil e envolve diversos fatores econômicos, políticos e culturais, sendo que a automedicação pode apresentar-se de forma benéfica ou não para a pessoa. Assim, é essencial que a educação e a sensibilização dos profissionais de saúde e da população, com o objetivo de alcançar o uso racional de medicamentos já que o surgimento de efeitos adversos são comuns ao utilizar medicamentos, tais como a fluoxetina e a amitriptilina. Isso porque os antidepressivos tricíclicos (TCAs) foram os antidepressivos mais comumente usados $(76,4 \%)$, seguidos por inibidores da recaptação da serotonina (SSRIs) $(18,1 \%)$ e inibidores da monoamina oxidase (IMAO) (6,8\%) (SOUSA et al., 2018).

Outros autores relacionam o uso de antidepressivos durante a gestação com um maior risco de pré-eclâmpsia. Estas e outras alterações implicam que deve existir um maior controle na prescrição de tais medicamentos, bem como um controle mais constante dos pacientes em uso desse tipo de medicação (PALMSTEN et. al., 2012). Os ADTs também são contraindicados em pessoas que possuem prostatismo ou arritmias (SCALCO, 2002). Especialmente nos idosos, deve-se levar em consideração que eles costumam apresentar diversas doenças simultaneamente, o que leva à utilização concomitante de diversos medicamentos. Além disso, ocorrem mudanças tanto na função renal quanto na composição corporal desses idosos ocasionadas pelo processo de envelhecimento, o que pode, consequentemente, desencadear reações indesejadas devido às interações medicamentosas já que essas interações podem gerar interferências farmacocinéticas e farmacodinâmicas nos remédios (GORZONI, FABBRI e PIRES, 2012). No mais, os agentes antidepressivos, assim como outros medicamentos que atuam no sistema nervoso central requerem tratamento por período prolongado, sendo este de dois anos ou mais, e a maior sensibilidade desses indivíduos às reações adversas pode comprometer a sua adesão ou criar situações de risco (MARIN et al., 2008).

Deve-se atentar para prevenção de interações medicamentosas, as quais são comuns com a utilização concomitante dos ADTs com agentes anticolinérgicos, anticoagulantes, anestésicos, inibidores da monoaminaoxidade (IMAO), anticonvulsivantes, anti-hipertensivos, bloqueadores histamínicos H2, levodopa, quinidina, roserpina e aminas simpatomiméticas. Além de serem contraindicados em pacientes que possuem glaucoma de ângulo fechado, bloqueios de ramo esquerdo, bloqueio $\mathrm{AV}$ total, íleo paralítico, hiperplasia prostática benigna (HPB), alterações na condução intracardíaca e infarto agudo do miocárdio (MORENO; MORENO; SOARES, 1999). Quanto à intoxicação por superdosagem, os seguintes sintomas podem ocorrer: confusão mental, convulsões, alterações de concentração, sonolência grave, alargamento de pupilas, alteração da frequência cardíaca, febre, alucinações, inquietação ou agitação, respiração curta ou difícil, cansaço e fraqueza intensa e vômitos. O tratamento da intoxicação consiste na tentativa de diminuir a absorção seja por esvaziamento gástrico seja com lavagem gástrica e na tentativa de aumentar a eliminação por meio da administração de pasta de carvão ativado seguida de estimulação catártica, além do tratamento de suporte necessário mediante as intercorrências cardiopulmonares (MORENO; MORENO; SOARES, 1999). Já a síndrome de abstinência ou de descontinuação ocorre em um pequeno 
grupo de pacientes que realizam a interrupção abrupta dos ADTs, especialmente após a realização de um tratamento prolongado. Essa síndrome costuma ocorrer nas primeiras 48 horas após a suspensão do antidepressivo e os sintomas podem estar relacionados a um efeito rebote de hiperatividade colinérgica, como por exemplo: mal-estar geral, alterações gastrintestinais (náuseas, vômitos e diarreia), ansiedade, irritabilidade, insônia, sonhos vívidos, movimentos parkinsonianos ou acatisia, além de poderem acontecer ataques de pânico, arritmias cardíacas e delirium. Logo, a recomendação é que a dose da medicação seja diminuída de maneira gradual ao longo de algumas semanas (MORENO; MORENO; SOARES, 1999).

É necessário relatar a importância do desenvolvimento de novos estudos referentes às vias receptoras pós-sinápticas e de mensageiros secundários, além da avaliação da expressão genética, pois estes podem ajudar a elucidar quais são as modificações que acontecem em um prazo mais longo no funcionamento do cérebro como resultado do uso crônico de antidepressivos (RANG, 2012). Devido à tudo que foi explicitado, os profissionais de saúde estão aceitando a utilização de antidepressivos mais recentes, como os ISRS, por estes serem mais seletivos e consequentemente apresentarem menos efeitos colaterais, além de serem bem tolerados e seguros, especialmente em casos de sobredosagem (FILHO et al., 2014). No entanto, pelo fato de todos os ADT possuírem eficácia comprovada no tratamento da depressão e da ansiedade, por em doses mais altas serem utilizados para tratar a dor e por conseguirem melhorar também o sono, como é o caso da amitriptilina, estes medicamento ainda são amplamente utilizados no dia a dia da clínica médica (DWORKIN et al., 2007). Portanto, fazse necessário dizer que os ADTs demonstram ser o padrão-ouro no tratamento da depressão grave e recorrente pela enorme eficiência que possuem como antidepressivos, tornando-se o medicamento preferido dos psiquiatras para tratar esses quadros. No mais é importante ressaltar que a utilização dos antidepressivos tricíclicos tem benefícios e eficácia comprovada e pode ser realizada de maneira segura se os pacientes forem monitorizados de maneira adequada (SCALCO, 2002).

\section{CONCLUSÃO}

A revisão apresentada demonstra que os antidepressivos tricíclicos são medicamentos amplamente utilizados na clínica médica devido ao custo benefício que apresentam, uma vez que eles são remédios de fácil acesso, disponíveis no SUS e que possuem uma larga utilização, já que tratam de maneira eficiente diversas patologias bastante prevalentes na medicina atual. No entanto, a presente revisão também sugere que os $\mathrm{ADT}$ possuem um alto risco toxicológico e diversos efeitos colaterais, presentes, especialmente, quando utilizados em idosos ou em pacientes com comorbidades, em doses altas ou de forma concomitante com outros medicamentos, por possuírem uma significativa interação medicamentosa com diversas classes de remédios. Sendo, assim, é importante destacar que essas reações adversas podem ser minimizadas ou até mesmo evitadas, desde que os medicamentos sejam utilizados de maneira cautelosa, com monitoramento constante dos pacientes e nas doses mais baixas possíveis para se obter o efeito desejado com o mínimo de efeitos colaterais.

\section{REFERÊNCIAS}

BRUNO, Marco AD; KRYMCHANTOWSKI, Abouch V. Amitriptyline and intraoral devices for migraine prevention: a randomized comparative trial. Arquivos de neuro-psiquiatria, v. 76, n. 4, p. 213-218, 2018.

CAMPIGOTTO, Kassia Fernanda et al. Deteç̧ão de risco de interações entre fármacos antidepressivos e associados prescritos a pacientes adultos. Archives of Clinical Psychiatry (São Paulo), v. 35, n. 1, p. $1-5,2008$.

CUNHA JR, Ricardo Joaquim da; BARRUCAND, Louis; VERÇOSA, Nubia. Estudo das alterações eletrocardiográficas com o uso de antidepressivos tricíclicos em pacientes com dor crônica. Revista Brasileira de Anestesiologia, v. 59, n. 1, p. 46$55,2009$.

DWORKIN, Robert $H$. et al. Pharmacologic management of neuropathic pain: evidence-based recommendations. Pain, v. 132, n. 3, p. 237-251, 2007.

ESTEVES, Fernanda Cavalcante; GALVAN, Alda Luiza. Depressão numa contextualização contemporânea. Aletheia, n. 24, p. 127$135,2006$.

GORZONI, Milton Luiz; FABBRI, Renato Moraes Alves; PIRES, Sueli Luciano. Medicamentos potencialmente inapropriados para idosos. Revista da Associação Médica Brasileira, v. 58, n. 4, p. 442-446, 2012.

LOPES, Claudia S.; FAERSTEIN, Eduardo; CHOR, Dóra. Eventos de vida produtores de estresse e transtornos mentais comuns: resultados do Estudo Pró-Saúde. Cadernos de Saúde Pública, v. 19, n. 6, p. 1713-1720, 2003.

LOPES, Diou David da Silva. O consumo de psicoativos: analise da psicofarmacologia de antidepressivos. FACIDER-Revista Científica, n. 7, 2015.

LOYOLA FILHO, Antônio Ignácio de et al. Tendências no uso de antidepressivos entre idosos mais velhos: Projeto Bambuí. Revista de Saúde Pública, v. 48, p. 857-865, 2014.

MARIN, Maria José Sanches et al. Caracterização do uso de medicamentos entre idosos de uma unidade do Programa Saúde da Família. Cadernos de Saúde Pública, v. 24, p. 1545-1555, 2008.

MORENO, Ricardo Alberto; MORENO, Doris Hupfeld; SOARES, Márcia Britto de Macedo. Psicofarmacologia de antidepressivos. Brazilian Journal of Psychiatry, v. 21, p. 24-40, 1999.

OLIVEIRA JÚNIOR, José Oswaldo de; ALMEIDA, Mauro Brito de. The current treatment of fibromyalgia. BrJP, v. 1, n. 3, p. 255$262,2018$.

OLIVEIRA, Márcio Galvão et al. Consenso brasileiro de medicamentos potencialmente inapropriados para idosos. Geriatr, Gerontol Aging, v. 10, n. 4, p. 168-81, 2016.

PALMSTEN, Kristin et al. Elevated risk of preeclampsia in pregnant women with depression: depression or antidepressants?. American journal of epidemiology, v. 175, n. 10, p. 988-997, 2012.

RANG, H. P. et al. Rang\&Dale Farmacologia, $7^{\circ}$ Edição. 2012.

ROCHA, Bruno Simas da; WERLANG, Maria Cristina. Psicofármacos na Estratégia Saúde da Família: perfil de utilização, acesso e estratégias para a promoção do uso racional. Ciência \& Saúde Coletiva, v. 18, p. 3291-3300, 2013.

RODRIGUES, Maria Aparecida P.; FACCHINI, Luiz Augusto; LIMA, Maurício Silva de. Modificações nos padrões de consumo de psicofármacos em localidade do Sul do Brasil. Revista de Saúde Pública, v. 40, p. 107-114, 2006.

SCALCO, Mônica Z. Tratamento de idosos com depressão utilizando tricíclicos, IMAO, ISRS e outros antidepressivos. Brazilian Journal of Psychiatry, v. 24, p. 55-63, 2002.

SEBASTIÃO, Elza; PELÁ, Irene Rosemir. Consumo de psicotrópicos: análise das prescrições ambulatoriais como base para estudos de problemas relacionados com medicamentos. Pharmacy Practice, v. 2, n. 4, p. 250-266, 2004.

SINDRUP, Søren H. et al. Antidepressants in the treatment of neuropathic pain. Basic \& clinical pharmacology \& toxicology, v. 96 , n. 6, p. 399-409, 2005.

SOUSA, Livia Alves Oliveira de et al. Prevalência e características dos eventos adversos a medicamentos no Brasil. Cadernos de Saúde Pública, v. 34, p. e00040017, 2018.

STUMP, Patrick Raymond Nicolas André Ghislain; KOBAYASHI, Ricardo; CAMPOS, Alexandre Walter de. Lombociatalgia. Revista Dor, v. 17, p. 63-66, 2016.

VICENTE, Adriano Roberto Tarifa et al. Uso de antidepressivos e fatores associados em idosos: o Projeto Bambuí. Ciência \& Saúde Coletiva, v. 20, n. 12, p. 3797-3804, 2015. 